\title{
Schmerztherapie
}

\section{Dualer Wirkansatz beugt Chronifizierung vor}

Bei einer anhaltenden Stimulation der aufsteigenden Schmerzsignale und/oder einer Schwächung der absteigenden Schmerzhemmung kann es zur Chronifizierung von Schmerzen kommen. Beide Mechanismen inhibiert Tapentadol (Palexia ${ }^{\circledR}$ retard) zentral als $\mu$-Opioid-Rezeptor-Agonist und als Noradrenalin-Wiederaufnahmehemmer am Rückenmark.

Die gute Wirksamkeit und Verträglichkeit von Tapentadol retard wurde in Studien zu rein nozizeptiven (Arthrose [Steigerwald I et al. 2012]) oder rein neuropathischen Schmerzen (diabetische Polyneuropathie [Schwartz S et al. 2011] und auch bei gemischten Schmerzen (vor allem chronischen Rückenschmerzen) belegt.

In einer aktuellen Vergleichsstudie reduzierte Tapentadol die Schmerzintensität bei 117 Patienten mit starken chronischen Rückenschmerzen mit neuropathischer und nozizeptiver Schmerzkomponente um 3,7 Punkte signifikant stärker als die Fixkombination aus retardiertem Oxycodon/Naloxon ( $\mathrm{n}=112$; $2,7)$ und verursachte weniger gastrointestinale Nebenwirkungen wie Obstipation und Erbrechen berichtete Prof. Ralf Baron, Kiel.

\section{Lebensqualität steigt}

Retardiertes Tapentadol stellt in einer hochdosierten Monotherapie ebenso wie in Kombination mit Pregabalin eine effektive Therapie bei chronischen Rückenschmerzen mit neuropathischer Komponente dar. Dies zeigte eine doppelblinde Studie [Baron R et al. 2015], in der die Patienten über acht Wochen $500 \mathrm{mg} / \mathrm{d}$ Tapentadol retard $(\mathrm{n}=154)$ oder die Kombination 300 mg/d Tapen- tadol retard/300 mg/d Pregabalin ( 159) erhielten. Schmerzen, Angst und Depressionen nahmen in beiden Gruppen signifikant und vergleichbar ab, parallel dazu stiegen Lebensqualität und Funktionalität. Die Verträglichkeit war in beiden Gruppen gut. Signifikante Vorteile für die Tapentadolmonotherapie ergaben sich bei Schwindel und Somnolenz.

Tapentadol kann eine Schmerzchronifizierung verhindern, wenn es möglichst früh eingesetzt wird, so der Kieler Neurologe. Am meisten profitieren $\mathrm{Pa}$ tienten mit einem hohen Pain-DetectScore, einem geringen Angst-Score und eher wenigen Schmerzattacken.

Stephanie Kraus

- Lunch-Symposium „Facetten der modernen patientenzentrierten Schmerztherapie", Deutschen Schmerzkongress; Mannheim, Oktober 2015 (Veranstalter: Grünenthal)

\section{Atemwegsinfekte}

\section{Lieber Phytotherapeutika als Antibiotika}

Laut dem Gesundheitsreport 2015 der Techniker Krankenkasse sind Antibiotika mit knapp $11 \%$ aller Verordnungen die am häufigsten rezeptierte Medikamentenklasse. Diese hohe Verordnungsrate wird u. a. durch den Einsatz bei akuten Atemwegsinfektionen verursacht, obwohl es sich hierbei um Erkrankungen handelt, bei denen Antibiotika nicht unkritisch verwendet werden sollten. Im Gegensatz zu pflanzlichen Arzneimitteln wie Bronchipret ${ }^{\circledR}$ bringen Antibiotika bei Infektionen wie einer unkomplizierten Bronchitis nachweislich keinen Therapievorsprung.

\section{Sekretnormalisierung und Entzündungshemmung}

Die Effektivität von Bronchipret ${ }^{\circledR}$ beruht auf den sekretnormalisierenden und antiphlogistischen Effekten der enthaltenen Pflanzenextrakte (Thymian und Efeu im Saft; Thymian und Primel in
Filmtabletten). In einem In-vivo-Modell der Bronchoalveolitis reduzierten beide Präparate die Zunahme der Zahl schleimbildender Becherzellen. Auch weitere Parameter wie Entzündungsmediatoren in der bronchoalveolären Lavage-Flüssigkeit werden positiv beeinflusst.

Randomisierte, doppelblinde, placebokontrollierte klinische Studien belegen die sehr gute Wirksamkeit des Phytherapeutikums bei akuter Bronchitis. So lässt unter anderem die Zahl der Hustenanfälle signifikant schneller nach: Eine 50\%ige Reduktion der Hustenanfälle wird bereits nach 6 Tagen erreicht - ca. 2 Tage früher als unter Placebo [Kemmerich B et al. Arzneim Forsch Drug Res. 2006;56:652-60; Kemmerich B. Arzneim Forsch Drug Res. 2007;57: 607-15].

Diese Wirksamkeitsbelege führten dazu, dass das Präparat in der S3-Hustenleitlinie der Deutschen Gesellschaft

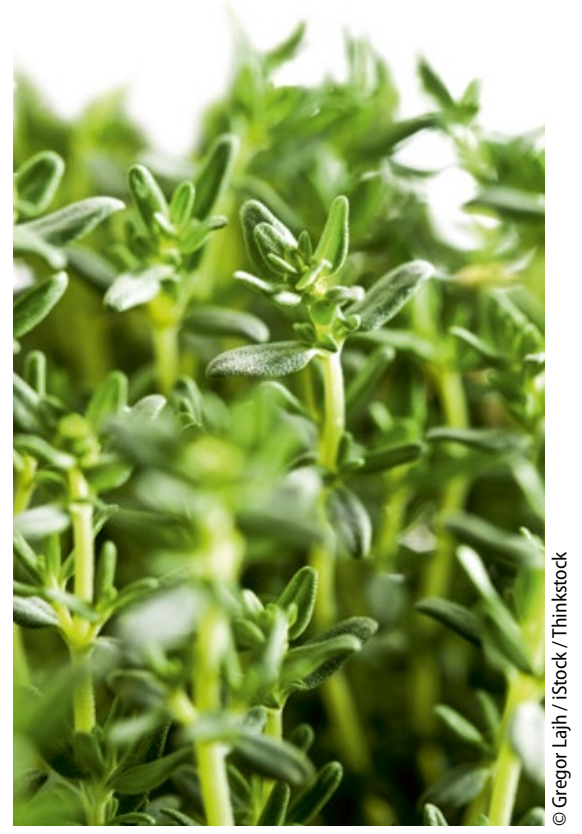

Thymian - ein Antiphlogistikum aus der Natur.

für Pneumologie und Beatmungsmedizin als einziges Medikament gegen akuten Husten empfohlen wird.

Red.

- Nach Informationen von Bionorica 